\title{
Generation of femtosecond paraxial beams with arbitrary spatial distribution
}

\author{
Ó. Martínez-Matos, ${ }^{1}{ }^{*}$ José A. Rodrigo, ${ }^{2}$ M. P. Hernández-Garay, ${ }^{1}$ J. G. Izquierdo, ${ }^{3}$ R. Weigand, ${ }^{1}$ \\ M. L. Calvo, ${ }^{1}$ P. Cheben, ${ }^{4}$ P. Vaveliuk, ${ }^{1,5}$ and L. Bañares ${ }^{3}$ \\ ${ }^{1}$ Departamento de Óptica, Facultad de Ciencias Físicas, Universidad Complutense de Madrid, 28040, Madrid, Spain \\ ${ }^{2}$ Instituto de Óptica (CSIC), Imaging and Vision Department, Serrano 121, Madrid 28006, Spain \\ ${ }^{3}$ Departamento de Química Física I, Facultad de Ciencias Químicas, \\ Universidad Complutense de Madrid, 28040, Madrid, Spain \\ ${ }^{4}$ Institute for Microstructural Sciences, National Research Council of Canada, KlA OR6 Ottawa, Canada \\ ${ }^{5}$ Faculdade de Tecnologia, SENAI-Cimatec, Av. Orlando Gomes 1845, 41650010 Salvador, BA, Brazil \\ *Corresponding author: omartine@fis.ucm.es
}

Received October 26, 2009; revised December 30, 2009; accepted January 19, 2010; posted January 25, 2010 (Doc. ID 119019); published February 22, 2010

We present an approach to generate paraxial laser beams with arbitrary spatial distribution in the femtosecond time regime. The proposed technique is based upon a pair of volume phase holographic gratings working in parallel arrangement. It exploits the spatial coherence properties of the incoming laser beam in a compact and robust setup that mitigates angular and spatial chirp. The gratings were recorded in a photopolymerizable glass with a high optical damage threshold and a large optical throughput. Setup performance is studied and experimentally demonstrated by generating Laguerre-Gaussian femtosecond pulses. (C) 2010 Optical Society of America

OCIS codes: $050.1950,090.2890,090.7330,090.1760,140.3300,160.6060,320.7090$.

The generation of arbitrary paraxial beams in the femtosecond time regime is a matter of great interest in fundamental and applied ultrafast sciences. The control of the spatial distribution of the wavefront, amplitude, and phase offers an additional degree of freedom in pulse shaping that open new insights in light-matter interaction. This is relevant for various applications, including nonlinear spectroscopy and microscopy, selective femtochemistry, and optical communications [1]. In spite of the potential applications of pulse spatial distribution control, only a few examples are found in the literature, many of them related to the creation of optical vortex beams.

The challenge in creating a particular spatial wavefront distribution in femtosecond laser pulses, with broad spectral bandwidths and preserving the temporal regime, is to impose such distribution onto all spectral components without angular and spatial chirp. Grating-based shapers provide large bandwidth operation and hence are suitable for ultrashort pulse manipulation. Specifically, the angular dispersion arising from diffraction in the first grating is compensated by a second grating with the same period, after the sign of the angular dispersion is reversed with an imaging optical system: lens [2-4] or curved mirrors [5] in a $4 f$ or $2 f-2 f$ setup, as depicted in Fig. 1(a). Several modifications of these setups have been recently reported [6-8]. The first grating operates as a dispersive element, while the second grating is used to encode a specific spatial distribution. The operational principle of such setups is based on the properties of imaging optical systems that establish a point-to-point correspondence between the gratings G1 and G2; see Fig. 1(a).

In this Letter we propose a method to generate paraxial beams in the femtosecond time regime exploiting the properties associated to the incoming beam, which has the same spectral content at each point of its transversal section and is spatially coherent. The proposed setup consists of a pair of volume phase holographic gratings (VPHGs) with the same spatial period, in a parallel arrangement and separated by a distance $L$, see Fig. $1(\mathrm{~b}) . \mathrm{G} 1$ is a plane grating, whereas the grating G2 encodes the beam to be generated. Light diffracted by G1 illuminates G2 affected by an angular and spatial chirp originated by dispersion in G1 and by the free-space propagation of the beam between the two gratings. From

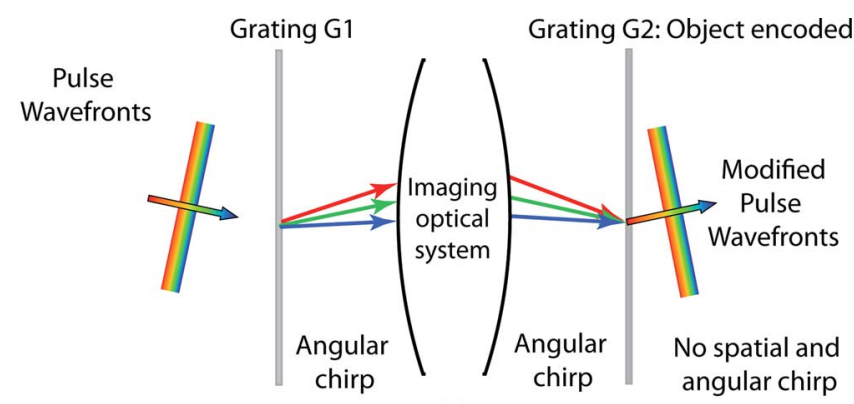

(a)

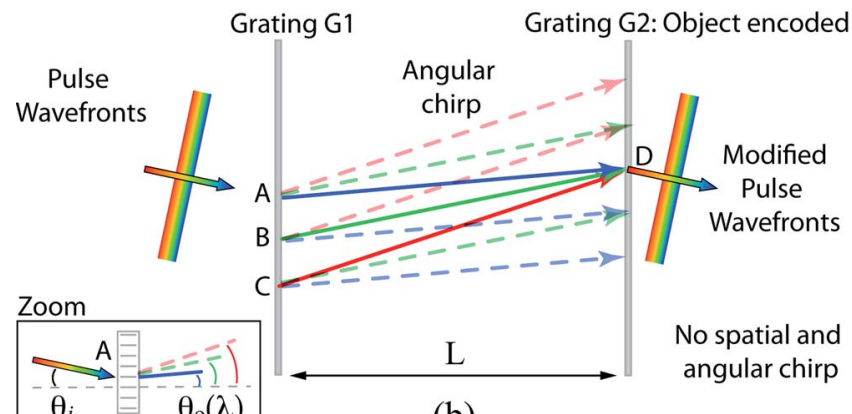

(b)

Fig. 1. (Color online) (a) Schematic of the femtosecond beam shapers previously reported. In contrast to such grating-based shapers, our setup (b) does not require an additional imaging optical system. Rays that do not intercept point $\mathrm{D}$ are displayed as dashed arrows. 
each point in G1 (e.g., A, B, and C) emerges a set of nonparallel rays whose wavelengths in the spectrum define their slopes following the Bragg condition $\sin \vartheta_{i}+\sin \vartheta_{o}=\lambda / d$, where $\vartheta_{i}, \vartheta_{o}$, and $d$ are the incident angle for the whole spectrum, the output angle for each wavelength $\lambda$, and the grating period, respectively. Only one ray from A impinges on the point D on grating G2, as shown in Fig. 1(b). In the same way, points $\mathrm{B}$ and $\mathrm{C}$ contribute in $\mathrm{D}$ with their corresponding wavelengths. To simplify visualization the rays that do not intercept point $\mathrm{D}$ are displayed as dashed arrows. This fact is extended to the overall plane containing G1, and therefore the whole spectrum of the input pulse is recovered at point $\mathrm{D}$. In an analogous way all points in G2 receive the contributions of all points in G1, also recovering the whole spectrum. This is a mandatory condition. Taking into account the finite width of the incoming beam, our approach is limited to a grating separation in which this condition is satisfied.

All spectral components diffracted in G2, e.g., at point D, acquire the same spatial information encoded in this location. Since both gratings have the same period, the angular chirp is suppressed, yielding collinear rays emerging from $\mathrm{D}$. Therefore the overall action of G2 imposes the spatial distribution of the signal onto all spectral components without angular and spatial chirp.

The spatial coherence of the incident beam assures a well-defined phase correlation among the points $\mathrm{A}$, $\mathrm{B}$, and $\mathrm{C}$ on the input wavefront and therefore a welldefined temporal correlation among the beams impinging point $\mathrm{D}$. This property is maintained after diffraction in grating G2, leading to spectral phase correlation of the outgoing beam. The temporal pulse character is preserved in point $\mathrm{D}$ and, by extension, in the overall plane containing G2. In conclusion, the spatial coherence of the incoming beam allows the design of an optical system in which it is not necessary to establish a point-to-point correspondence between gratings G1 and G2 to obtain arbitrary spatial distribution of the paraxial beams. This lensless setup substitutes the imaging optical system previously reported, and hence, resulting in a compact and robust optical setup. To the best of our knowledge, this is the first time that properties inherent to the spatial coherence of light have been applied to spatial pulse shaping, opening a new degree of freedom when generating different types of paraxial beams.

The gratings G1 and G2 implemented in our system are volume transmission phase gratings holographically recorded in a photopolymerizable glass [9-12]. This material is a modified composition of a highly efficient photopolymerizable sol-gel glass synthesized earlier by Cheben and Calvo [13], wherein the modification is achieved by incorporating the $\mathrm{Zr}$ based high refractive index species at molecular level [9]. Grating formation is obtained by two mutually coherent $s$-polarized writing beams using a cw frequency-doubled Nd:YAG laser at $532 \mathrm{~nm}$. Recording mechanism of the grating and its optical properties have been studied elsewhere for $\mathrm{cw}$ lasers [9-12], demonstrating an excellent optical performance. In this work we implement gratings recorded in this material as holographic optical element for femtosecond pulse lasers.

Both gratings, G1 and G2, were recorded with a spatial frequency of 500 lines $/ \mathrm{mm}$ by interfering two writings beams $(532 \mathrm{~nm})$ at a half-angle $\theta=7.9^{\circ}$. The G1 is an unslanted plane grating, whereas the G2 encodes the signal. To demonstrate the optical performance of the proposed setup, we recorded several paraxial beams on grating G2. In particular here we consider a Laguerre-Gaussian (LG) mode $\mathrm{LG}_{p, l}$, where $p$ and $l$ are the radial index and topological charge, respectively (e.g., see [8] and references there in). An LG mode is a well-known example of optical vortex $(l \neq 0)$ that carries an orbital angular momentum of $l \hbar$ per photon. Such signals are important for various fields of research and practical applications, e.g., they are used to investigate orbital angular momentum effects on light-matter interactions, optical trapping, and quantum computing, to name a few. There are different methods for the generation of these signals, including computer generated holograms [14]. In our case we use a programmable optical setup [15] able to generate arbitrary scalar paraxial beams by using two spatial light modulators (SLMs), as in Fig. 2(a). The amplitude, Fig. 2(b), and phase distribution, Fig. 2(c), of the complex signal are addressed onto SLM1 and SLM2, respectively. The image displayed in SML1 is projected (by using a $4 f$ setup) into SLM2, yielding the desired output beam (e.g., an LG mode). This output beam is holographically recorded in the photopolymerizable glass (HM), as shown in Fig. 2(a), where a collimated reference beam is used. The $\mathrm{LG}_{1,2}$ mode displayed in Fig. 2(d) was reconstructed from the hologram by using a cw collimated readout beam at $532 \mathrm{~nm}$.

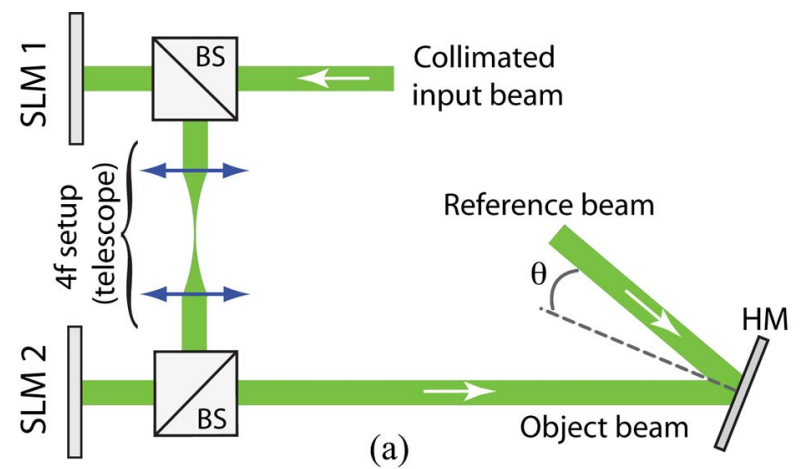

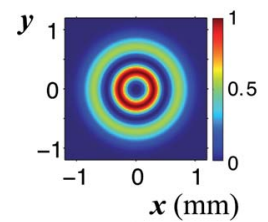

(b)

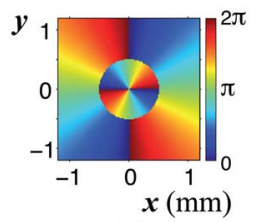

(c)

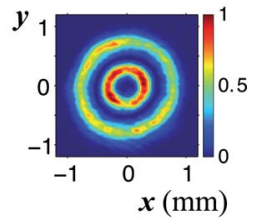

(d)
Fig. 2. (Color online) (a) Optical setup for arbitrary scalar paraxial beam creation. Amplitude and phase distribution are addressed onto SLM1 and SLM2 (Holoeye LCR-2500), respectively. (b) Intensity and phase distribution (c) of the considered $\mathrm{LG}_{1,2}$ beam (units are in millimeters). The resulting beam is recorded in the holographic material (HM). (d) Reconstructed holographic image captured by a CCD camera. 
The proposed setup was tested with pulses from a Ti:sapphire laser amplifier centred at $800 \mathrm{~nm}$, Gaussian shaped with $6 \mathrm{~mm}$ FWHM, with $3.6 \mathrm{~mJ}$ per pulse at $1 \mathrm{kHz}$ repetition rate, and positively chirped with $60 \mathrm{fs}$ duration. The gratings recorded in the photopolymerizable glass maintains the diffraction efficiencies after more than one million pulses and did not show any damage (tested by optical inspection using phase-contrast microscopy), demonstrating an excellent damage threshold.

To evaluate the setup performance we have studied the propagation of LG beams for near- and far- field regimes. Here we considered gratings separated by $L=300 \mu \mathrm{m}$. Notice that LG beams are structurally stable under free-space propagation, which has to be tested. As is demonstrated in Figs. 3(a) and 3(b) the $\mathrm{LG}_{1,2}$ pulsed beams are well defined and stable in near- as well as far-field propagation, respectively. This test has also been performed for other beams, proving their stability. For example, the propagation of $\mathrm{LG}_{0,6}+\mathrm{LG}_{0,-6}$ beam is displayed for near-field and far-field in Figs. 3(c) and 3(d), respectively. To achieve the far-field propagation we used a convergent spherical lens, in our case with focal distance of $20 \mathrm{~cm}$ and located just after the setup. These experimental results confirm a well-defined beam distribution, which implies that the VPHGs setup does not produce angular and spatial chirp.

This optical system is dispersive, and the temporal width of the outgoing pulsed beam depends on the distance $L$. Pulse durations at the exit of the G2 were measured with a commercial second-harmonic autocorrelator obtaining $90 \mathrm{fs}$ for $L=300 \mu \mathrm{m}, 156 \mathrm{fs}$ for $L=8 \mathrm{~mm}$ and $3 \mathrm{ps}$ for $L=20 \mathrm{~cm}$. This provides evi-

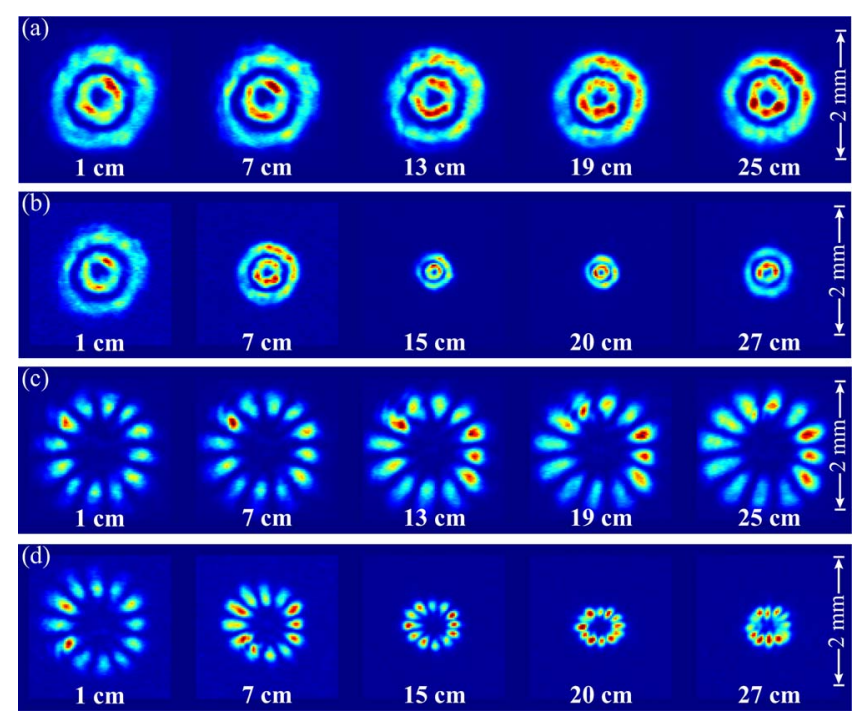

Fig. 3. (Color online) Free-space propagation of femtosecond pulsed beams generated with the VPHGs setup. (a) Near-field (b) and far-field propagation of $\mathrm{LG}_{1,2}$ beam is displayed. The $\mathrm{LG}_{0,6}+\mathrm{LG}_{0,-6}$ beam propagation in (c) nearfield and (d) far-field regimes. Labels in the figure indicates propagation distances. dence that pulses remained ultrashort for the most compact setups. The setup efficiency per unit area is close to $60 \%$ in the whole range of separations analyzed. In future research the temporal properties of the generated beams will be studied in detail, and the capacity of our optical system to compress pulses will be explored.

In this Letter we have presented a method to generate arbitrary scalar paraxial beams in the femtosecond regime exploiting the spatial coherence of the input beam. The proposed setup does not implement any imaging optical system, resulting in a compact, robust, and easy to align optical setup. Specifically, the setup employs two parallel volume phase gratings recorded in a photopolymerizable glass that exhibit a high optical throughput and very high optical damage threshold in femtosecond regime. These advantages indicate that the proposed technique is particularly promising for intense-field and ultrashort pulse applications.

We thank Fransisco del Monte for valuable advice and CAI-UCM facilities. The financial support from the Spanish Ministry of Science and Innovation under projects TEC 2008-04105, CTQ2008-02578/BQU and Consolider SAUUL CSD2007-00013 is acknowledged. M. P. H.-G. and P. V. acknowledge the Spanish Ministry of Foreign Affairs and Conselho Nacional de Desenvolvimento Científico e Tecnológico (CNPq) (Brazil) for financial support, respectively.

\section{References}

1. Claude Rullière ed., Femtosecond Laser Pulses: Principles and Experiments (Springer, 2005).

2. K. Bezuhanov, A. Dreischuh, G. G. Paulus, M. G. Schtzel, and H. Walther, Opt. Lett. 29, 1942 (2004).

3. I. G. Mariyenko, J. Strohaber, and C. J. G. J. Uiterwaal, Opt. Express 13, 7599 (2005).

4. I. J. Sola, V. Collados, L. Plaja, C. Mndez, J. San Romn, C. Ruiz, I. Arias, A. Villamarín, J. Atencia, M. Quintanilla, and L. Roso, Appl. Phys. B 91, 115 (2008).

5. I. Zeylikovich, H. I. Sztul, V. Kartazaev, T. Le, and R. R. Alfano, Opt. Lett. 32, 2025 (2007).

6. A. Schwarz and W. Rudolph, Opt. Lett. 33, 2970 (2008).

7. K. Bezuhanov, A. Dreischuh, G. G. Paulus, M. Schtzel, H. Walther, D. Neshev, W. Królikowski, and Y. Kivshar, J. Opt. Soc. Am. B 23, 26 (2006).

8. J. Strohaber, C. Petersen, and C. J. G. J. Uiterwaal, Opt. Lett. 32, 2387 (2007).

9. F. del Monte, O. Martínez-Matos, J. A. Rodrigo, M. L. Calvo, and P. Cheben, Adv. Mater. 18, 2014 (2006).

10. O. Martínez-Matos, M. L. Calvo, J. A. Rodrigo, P. Cheben, and F. del Monte, Appl. Phys. Lett. 91, 1 (2007).

11. M. L. Calvo and P. Cheben, J. Opt. A 11, 024009 (2009).

12. O. Martnez Matos, J. A. Rodrigo, M. L. Calvo, and P. Cheben, Opt. Lett. 34, 485 (2009).

13. P. Cheben and M. L. Calvo, Appl. Phys. Lett. 78, 1490 (2001).

14. J. A. Davis, D. M. Cottrell, J. Campos, M. J. Yzuel, and I. Moreno, Appl. Opt. 38, 5004 (1999).

15. J. A. Rodrigo, T. Alieva, and M. Calvo, J. Opt. Soc. Am. A 24, 3135 (2007). 